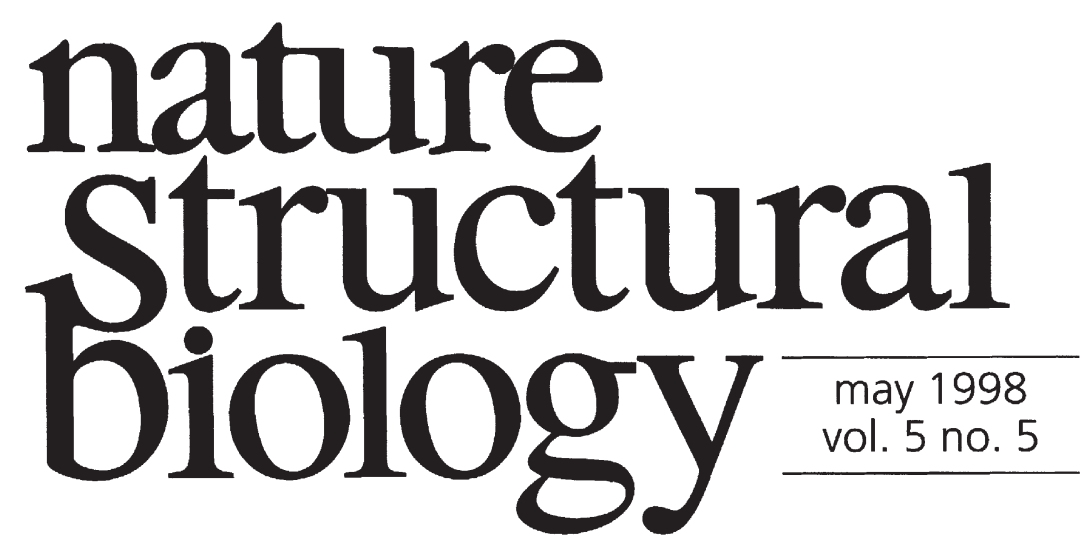

\title{
Fold me deadly
}

The consequences of failed protein folding reactions may be profoundly damaging and, in many cases, fatal. The deposition of normally soluble proteins in our body tissues as insoluble fibrils is thought to result in the structural and functional disruption of those tissues, a condition known as amyloidosis. Amyloidosis is closely associated with conditions such as Alzheimer's disease, Huntingtons' related diseases, and the prion-transmissible spongiform encephalopathies. Understanding the basis for the aberrant folding in such pathological conditions was merely one of the wide range of topics researchers grappled with at a recent meeting on protein folding*. What follows is a necessarily highly selective review of the proceedings.

The amyloid fibrils characteristic of different diseases are derived from proteins that bare little or no structural or functional relationship to each other. Nevertheless, all the fibrils have a similar structure, being $\sim 60-120 \AA$ in diameter, unbranched, and having features that suggest they consist of a helical cross- $\beta$-sheet arrangement of polypeptide chains parallel to the long axis of the fiber, with the $\beta$-strands perpendicular to this axis.

An understanding of the basis of fiber formation and persistence will be vital in developing strategies for dealing with these diseases. Jeff Kelly (Scripps Research Institute, USA) and Mark Pepys (Hammersmith Hospital, London, UK) provided examples of researchers who are tackling the problem from different ends: Kelly is working on ways to prevent the formation of the fibers, while Pepys is tackling the problem of how to disrupt fibrils that have already been deposited.

The fibrils in familial amyloid polyneuropathy and senile systemic amyloidosis are derived from transthyretin (TTR). TTR acts as a carrier for thyroxin (T4) in the blood and is normally found as a tetramer. Tetrameric TTR is not amyloidogenic: the substrate for fiber formation seems to be a conformational intermediate derived from the TTR monomer, formed by partial acid denaturation (which probably occurs in the lysosome in vivo, where the $\mathrm{pH}$ is $\sim 5$ ). Kelly has observed that binding of the T4 ligand stabilizes the tetrameric form of TTR against dissociation into the monomeric form, thereby almost completely preventing fibril formation in vitro. This result correlates with the observation that there is little incidence of TTR-based fiber formation in the brain, where TTR is the primary T4 carrier, whereas only $10-25 \%$ of the TTR in the blood plasma is bound to T4 (where T4 binding globulin is the primary carrier). In an effort to find tetramer-stabilizing ligands, Kelly has screened $\sim 5,000$ small molecules as potential drug leads and come up with two promising compounds: flufenamic acid (which is highly selective for TTR) and resveratrol, found in red wine.

The body has the capability of clearing amyloid fibrils in vivo, suggesting there is a fine balance between fibril formation and degradation: as a therapeutic strategy Pepys wants to tip the balance in favor of degradation. To this end he has been studying serum amyloid P component (SAP). SAP is an important part of the innate immune system, as well as being a DNA/chromatin binding protein. Bizarrely (and disastrously) SAP has also been found to coat all amyloid fibers, disastrously because binding of SAP (which is itself protease resistant) renders the fibers highly resistant to protease treatment in vitro, and may therefore contribute to the persistence and pathogenesis of the

${ }^{\star}$ EMBO Workshop - Protein folding and misfolding inside and outside the cell, 25-28 March, 1998, Oxford, UK. fibers in vivo. In support of this, SAP- mice show delayed and reduced fibril formation, suggesting that SAP is indeed a potential therapeutic target. Like Kelly, Pepys has been fishing for drugs and has come up with compound "R" (derived from a screen of a Roche small molecule library) that has the ability, in vitro at least, to strip SAP out of amyloid. 
Kinetics are critical: aggregates can take days to form whereas the regular folding pathway is often completed in seconds or less. The rates of these different processes are critical to the final fate of the protein. Chris Dobson
Amyloidogenic proteins presumably share some subtle biophysical properties that make them susceptible to this particular type of aggregation. Surprisingly, an $\mathrm{SH} 3$ domain from the p85 subunit of bovine phosphatidyl inositol-3 kinase can be induced to form fibrillar aggregates at very low $\mathrm{pH}$, which have biochemical properties similar to those of amyloid fibers. The structure of the SH3 fibrils has been determined by cryo-electron microscopy (José Luis Jiménez, Birkbeck College, London, UK). The individual fibers are hollow, with several protofilaments twisting around a central cavity. Although the fibers themselves are probably not physiologically relevant, the structural simplicity of the SH3 domain should a make this a valuable model for understanding fiber formation in vivo.

Of the broad swath of research into protein folding presented at the meeting (much more than could reasonably covered here) one area being tackled by researchers is the dissection of the substrate binding functions of many of the chaperones, to provide both insight into mechanism and useful biochemical tools.

Although protein disulfide isomerase (PDI), an endoplasmic reticulum (ER) resident chaperone, was the first catalyst of protein folding to be identified, little is known about how the protein interacts with its wide range of substrates. PDI is constructed from four structural domains with homologous thioredoxin folds, plus an acidic C-terminal extension (a-b-b'-a'-c). Robert Freedman (University of Kent, UK) reported that the $b^{\prime}$ domain of the protein is necessary and sufficient of binding small (10-15 residue) peptides, and is necessary but not sufficient for binding of bigger ( 28 residue) peptides; these latter peptides require larger fragments of PDI. The smallest fragment capable of binding both small peptides and bigger substrates is the $b^{\prime}-a^{\prime}-c$ fragment of PDI, which was also the smallest fragment that showed efficient catalysis of disulfide bond rearrangement.

Johannes Buchner (Universität Regensberg, Germany) and Jason Young (Martinsried, Germany) are engaged in a similar dissection of hsp 90 , one of the most abundant heat shock proteins in the cytoplasm. The protein consists of conserved $\mathrm{N}$ - and $\mathrm{C}$-terminal domains connected by a charged linker. The two domains have separate and independent chaperone activities that differ in their substrate specificities. The $\mathrm{N}$ domain recognizes unstructured peptides, with a degree of specificity, and dissociation is ATP dependent. The $\mathrm{C}$ domain promiscuously binds partially folded proteins - that is, structured substrates - in an ATP independent manner and does not bind peptides. The dual nature of the chaperone activity of hsp 90 may explain its dual functions in vivo as both a specific chaperone (under normal conditions) and general chaperone (under conditions of stress).

No conference on protein folding would be complete without mention of GroEL (see also the News and Views in this issue on the thermosome). Of the many and various presentations, Alan Fersht (Cambridge University, UK) has taken a brutally reductionist approach in studying this complex molecular machine, dissecting the active center away from the rest of the molecule. A $16,700 M_{\mathrm{r}}$ fragment of the apical domain (the GroEL monomer has a mass of $\sim 60,000$ ), from residues 191-345, has efficient chaperone activity in facilitating the refolding of cyclophilin A and rhodanese. In contrast, Frank Weber (Martinsried, Germany) reported that although a similiar apical fragment (191-376) can retard the aggregation of unfolded rhodanese it does not facilitate its refolding in vitro or in the presence of GroEL/ES, suggesting that, one way or the other, care should be taken when carving up proteins. Nevertheless, using his 'mini-chaperones', Fersht has developed a refolding chromatography protocol that has proven to be highly effective in solublizing 'insoluble' protein aggregates (but which has not, as yet, been universally successful). Furthermore, by using a combination of DsbA-linked beads with the mini-chaperone beads he reported the refolding of an 'insoluble' aggregate of a 66 amino-acid protein containing four disulfide bonds.

The end result of protein folding (ideally, and for good health) is a folded protein. Although there is much sterling working being done on the basic biophysical mechanism of folding, some of which was reported at the meeting, our insight into what has been dubbed the second half of the genetic code remains frustratingly vague. This has not deterred protein engineering efforts. Luis Serrano (EMBL, Heidelberg, Germany), for example, is using the simple strategy of stabilizing native-like secondary structure in designing proteins. By merely increasing the helical propensity of two helices in carboxypeptidase $\mathrm{A}$, he has been able to increase the melting temperature $\left(\mathrm{T}_{\mathrm{m}}\right)$ of the engineered protein to $\sim 105^{\circ} \mathrm{C}$, a gain of $35^{\circ} \mathrm{C}$ over the wild-type $\mathrm{T}_{\mathrm{m}}$. Using similar principals, he has also designed a three-stranded antiparallel $\beta$-sheet that is stable and monomeric in water (the 16-mer aggregates above $5 \mathrm{mg} \mathrm{ml}^{-1}$, but is monomeric at $3 \mathrm{mg} \mathrm{ml}^{-1}$ ). This peptide, which holds the record for being the smallest designed 'protein', has a $\Delta G$ of $4 \mathrm{kcal} \mathrm{mol}^{-1}$ at $40^{\circ} \mathrm{C}$, at which temperature it is $90 \%$ folded.

The question that these and other recent successful design efforts raise is, of what relevance is protein folding to protein engineering? It seems that the ability to fold is not one of the major constraints in the design of small proteins. Perhaps understanding folding will become more critical as protein engineers take on ever larger proteins. Indeed, such efforts may well provide fresh (and much needed) insights into the problem. 